\title{
Multimodal Mass Spectrometry Imaging of Rat Brain using IR-MALDESI and nanoPOTS-LC-MS/MS
}

Crystal L. Pace ${ }^{1}$, Jared Simmons ${ }^{2}$, Ryan T. Kelly², and David C. Muddiman ${ }^{1,3}$ ${ }^{1}$ FTMS Laboratory for Human Health Research, Department of Chemistry, North Carolina State University, Raleigh, NC, USA, ${ }^{2}$ Department of Chemistry and Biochemistry, Brigham Young University, Provo, UT, USA, ${ }^{3}$ Molecular Education, Technology and Research Innovation Center (METRIC), North Carolina State University, Raleigh, NC, USA

\section{Supporting Information}

\section{Table of Contents}

Figure S1 Additional examples of multiomic integration between metabolites and enzymes identified by IR-MALDESI and nanoPOTS-LC-MS/MS

Files

Table S1.xlsx Metabolites putatively annotated by IR-MALDESI with significant fold changes between ROI 4 and other analyzed ROls of the brain Table S2.xIsx Proteins identified in the rat brain by nanoPOTS-LC-MS/MS by Proteome Discoverer 2.4 

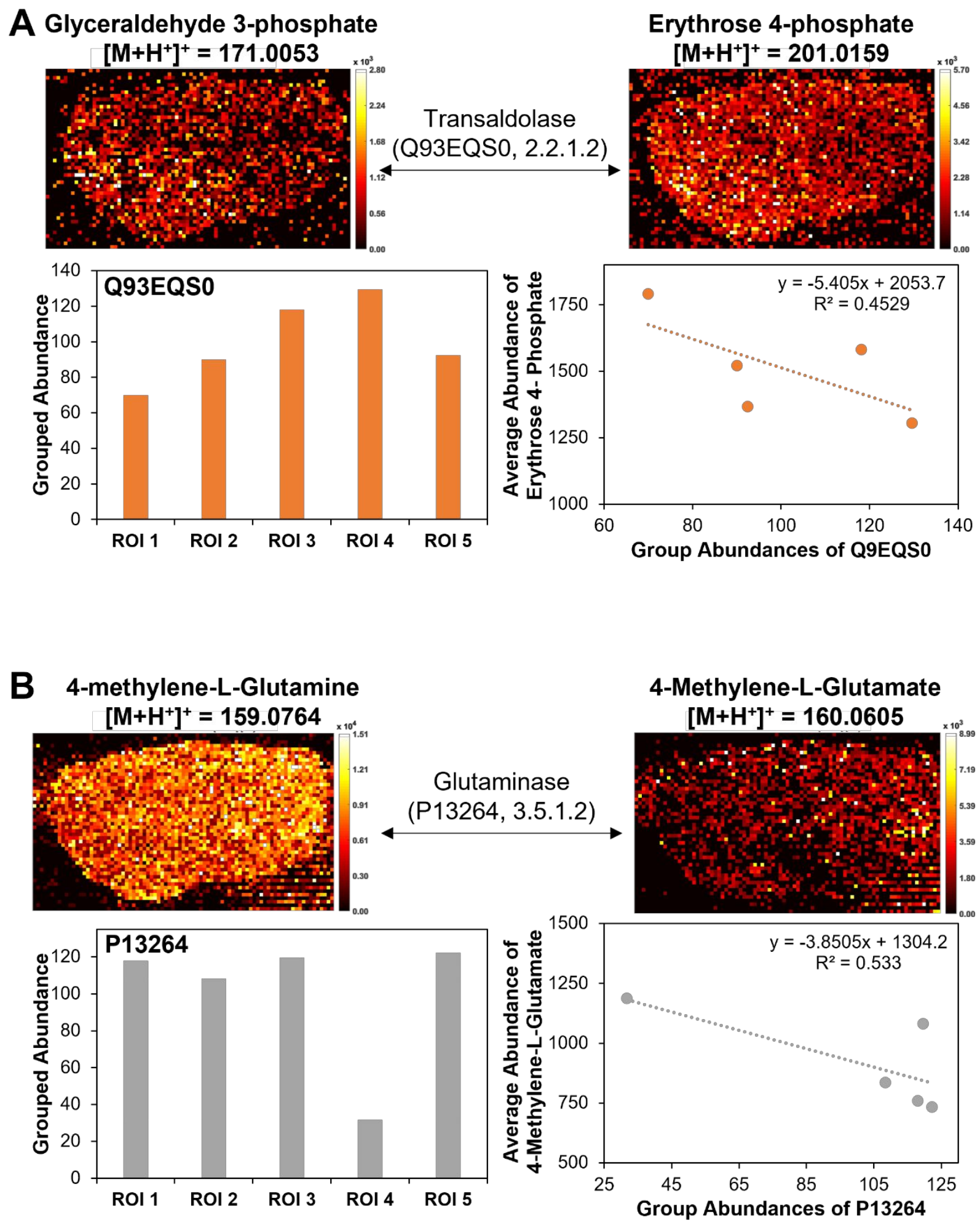


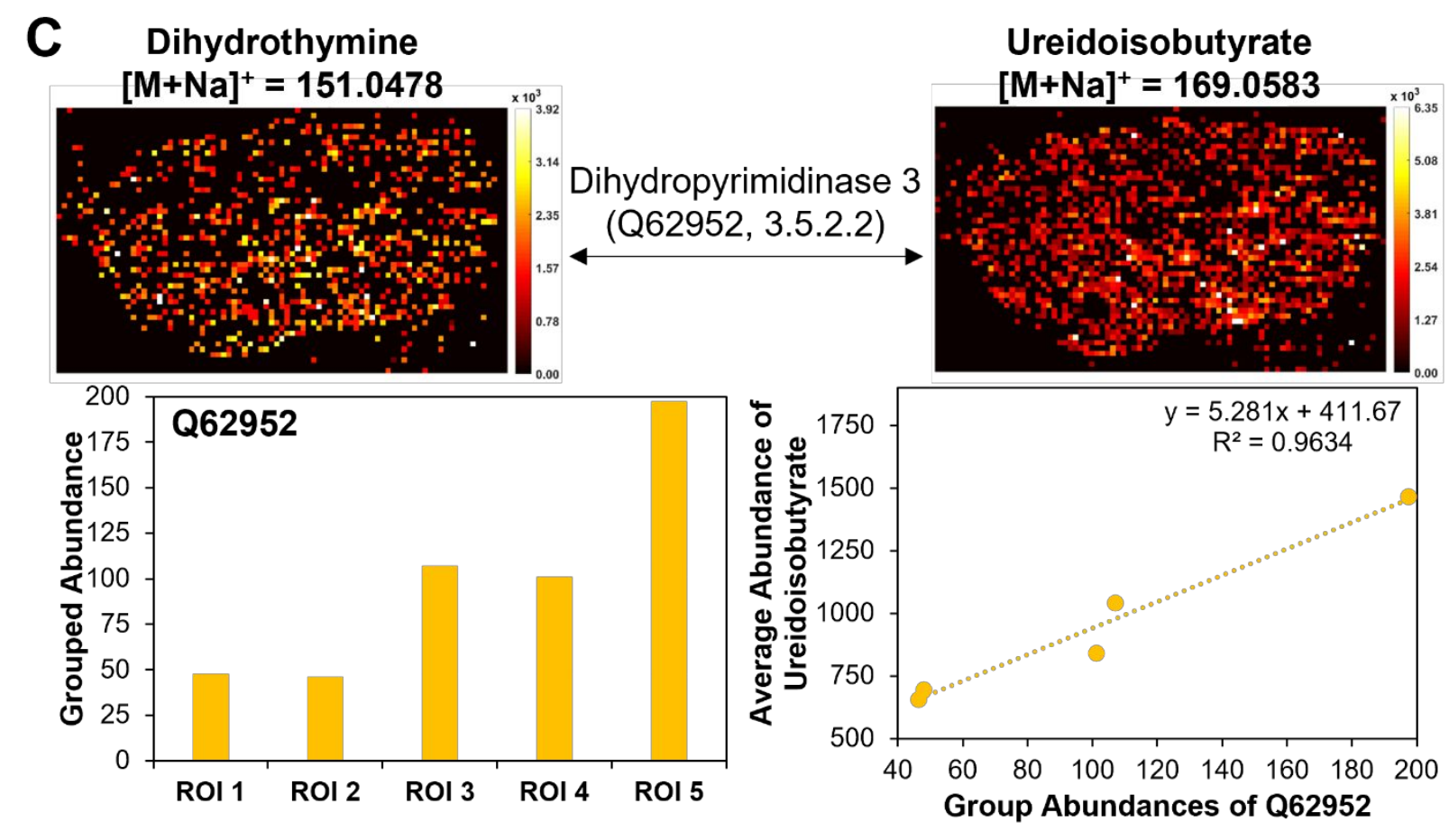

Figure S1. Additional examples of multiomic pathway integration of IR-MALDESI and nanoPOTS-LCMS/MS. Ion images represent metabolites detected by IR-MALDESI in the rat brain and the bar graph represents the average abundance of the enzyme involved in this metabolism for each brain region. Correlation plot of the grouped abundance of enzyme versus the average abundance of metabolite in each region show a strong or moderate correlation between the two datasets. 